\title{
Calibration of astigmatic particle tracking velocimetry based on generalized Gaussian feature extraction
}

\begin{abstract}
Simon Franchini, ${ }^{\mathrm{a}, \mathrm{b}}$ Alexandros Charogiannis, ${ }^{\mathrm{c}}$ Christos N Markides, ${ }^{\mathrm{c}}$ Martin J Blunt ${ }^{\mathrm{a}, \mathrm{b}}$ and Samuel Krevor ${ }^{a, b}$

Flow and transport in porous media are driven by pore scale processes. Particle tracking in transparent porous media allows for the observation of these processes at the time scale of ms. We demonstrate an application of defocusing particle tracking using brightfield illumination and a CMOS camera sensor. The resulting images have relatively high noise levels. To address this challenge, we propose a new calibration for locating particles in the out-of-plane direction. The methodology relies on extracting features of particle images by fitting generalized Gaussian distributions to particle images. The resulting fitting parameters are then linked to the out-of-plane coordinates of particles using flexible machine learning tools. A workflow is presented which shows how to generate a training dataset of fitting parameters paired to known out-of-plane locations. Several regression models are tested on the resulting training dataset, of which a boosted regression tree ensemble produced the lowest cross-validation error. The efficiacy of the proposed methodology is then examined in a laminar channel flow in a large measurement volume of 2048, 1152 and $3000 \mu \mathrm{m}$ in length, width and depth respectively. The size of the test domain reflects the representative elementary volume of many fluid flow phenomena in porous media. Such large measurement depths require the collection of images at different focal levels. We acquired images at 21 focal levels $150 \mu \mathrm{m}$ apart from each other. The error in predicting the out-of-plane location in a single slice of $240 \mu \mathrm{m}$ thickness was found to be $7 \mu \mathrm{m}$, while in-plane locations were determined with sub-pixel resolution (below 0.8 $\mu \mathrm{m})$. The mean relative error in the velocity measurement was obtained by comparing the experimental results to an analytic model of the flow. The estimated displacement errors in the axial direction of the flow were 0.21 pixel and 0.22 pixel at flows rates of $1.0 \mathrm{~mL} / \mathrm{h}$ and $2.5 \mathrm{~mL} / \mathrm{h}$, respectively. These results demonstrate that it is possible to conduct three-dimensional particle tracking in a representative elementary volume based on a simple apparatus comprising a microscope with standard brightfield illumination and a camera with CMOS sensor.
\end{abstract}

\section{Introduction}

\section{Motivation}

The area of flow in porous media relates to many processes which are driven by pore-scale velocities. Prominent examples are dispersion ${ }^{1,2}$ reactive transport ${ }^{3,4}$ and mass transfer ${ }^{5,6}$. Conventional measurement techniques used for the investigation of flow in porous media, such as X-ray computer tomography and positron emission tomography, achieve frame rates of $<1$ frame per second. To image dynamic pore scale processes such as Haines jumps, an ideal experimental apparatus must acquire images at the timescale of ms or less. ${ }^{7}$ Optical diagnostics are ideal for this purpose as they allow for the non-invasive visualization of two- and three-dimensional (2D and 3D) spatial distributions of tracers and particles over a wide range of timescales. However, their application relies on precise matching of the refractive indexes of fluids and solids. ${ }^{8,9}$

Particle image and particle tracking velocimetry (PIV and PTV) are now often employed towards the spatiotemporallyresolved flow-field characterization through the measurement

\footnotetext{
a. Department of Earth Science and Engineering, Imperial College London, London, UK.

b. Qatar Carbonates and Carbon Storage Research Centre, Imperial College London London, UK.

c. Clean Energy Processes (CEP) Laboratory, Department of Chemical Engineering, Imperial College London, London, UK.
}

of $2 \mathrm{D}$ or 3D velocity fields in a wide range of flows. In PIV, each velocity vector is associated with an area ('window') spanning a number of pixels and is thus based on the motion of particle groups. This improves accuracy but reduces resolution due to the effective filtering performed by the windowing process..$^{10}$ PTV, on the other hand, relies on the tracking of individual particles and has a higher resolution and a lower susceptibility to bias errors in the presence of strong velocity gradients such as in near-wall regions. ${ }^{11}$ Thus, PTV is often employed when investigating highly constrained flows and flows with high aspects ratios, such as thin-film flows. ${ }^{12-15}$ It is worth noting, however, that PTV only obtains velocity vectors at discrete (random) particle locations.

New micrometre-scale fabrication methods have enabled the manufacture of transparent 3D geometries with volumes in the order of $\mathrm{mm}^{3} .{ }^{16-18}$ Such large 3D geometries can generate complex flow phenomena both in lab-on-chip devices ${ }^{19,20}$ as well as in porous media with 3D connectivity. ${ }^{8,21-22}$ To reproduce the flow conditions that are observed in natural porous media, the depth of the device has to cover the length of a representative elementary volume (REV). ${ }^{23}$ Depending on the heterogeneity within the porous media, the length of the REV ranges from several 100s of $\mu \mathrm{m}$ up to a few $\mathrm{mm} .{ }^{24}$ Previous studies have demonstrated a method for tracking particles within this range using advanced microscopy, which either comprised custom-made optical arrangements, ${ }^{9,25}$ pulsed lasers for illumination as well as $\mathrm{CCD} / \mathrm{sCMOS}$ cameras 26,27 or confocal microscopy 8,28 . The primary aim of our 
study is to develop a technique which is capable of tracking the motion of particles in a volume which covers the REV of many flow phenomena in porous media using a microscope with conventional brightfield illumination and a camera with CMOS sensor.

\section{Comparison of 3D velocimetry techniques}

Several methods have been used to track the motion of particles in three dimensions at the micron scale. Over the last decade, a number of conventional 3D velocimetry techniques have found their way into the field of microfluidics. Examples include tomographic particle image velocimetry and 3D particle tracking velocimetry. ${ }^{9,29}$ These techniques, however, require optical access from multiple angles, and therefore, highly-customised optical arrangements. This limitation rules out the use of standard microscopes for image acquisition. Confocal microscopy has been successfully employed to obtain $2 \mathrm{D}$ velocity profiles through PIV and to image dynamic flow phenomena within porous media.8,28,30,31 This technique provides high spatial and temporal resolution in one plane; yet, a limitation of this method is that it is not able to acquire the velocity in the third dimension.

Defocusing particle tracking is a method which only requires optical access from one direction in order to obtain 3D particle coordinates. Therefore it can be employed using a standard microscope. The measurement principle is based on the reconstruction of the z-location of particles (i.e. their out-ofplane coordinate) directly from the respective particle images: particle images change shape with respect to their location to the focal plane of a microscope objective. There are two special variants which employ this measurement principle.

The first relies on the installation of a mask with three pinholes behind the microscope objective. ${ }^{25}$ This arrangement generates three images for each fluorescent particle on the camera sensor. The in-plane coordinates of each particle are found from the centre point of all three particle images, while the location of each particle in the out-of-plane direction is inferred from the distance between the three particle images. One of the challenges associated with this method is matching the multiple particle images to each particle. Thus, revised versions of this system rely on colour-coded pinholes and multiple light sources and cameras. ${ }^{32}$ The use of multiple cameras is a limitation, as it increases the cost of the equipment significantly, especially if the investigated flow requires high-speed, high-resolution visualisation.

The second defocusing method employs an astigmatic optical arrangement to create unique particle images along the $z$ direction. ${ }^{33}$ A cylindrical lens is placed behind the objective lens and ahead of the camera sensor, thus deforming the particle images in the $x$ and $y$ directions (i.e. along the in-plane coordinates). The resulting particle images resemble vertically or horizontally oriented ellipses. The orientation and shape of these ellipses depends on the location of the imaged particle with respect to the focal plane of the microscope objective (i.e. in the out-of-plane direction). By analysing the major and minor axes diameters of the particle images, the out-of-plane location of particles can be inferred. Several other methods can be used to determine the out-of-plane particle coordinates based on the analysis of image parameters. ${ }^{34,} 35$ In contrast to
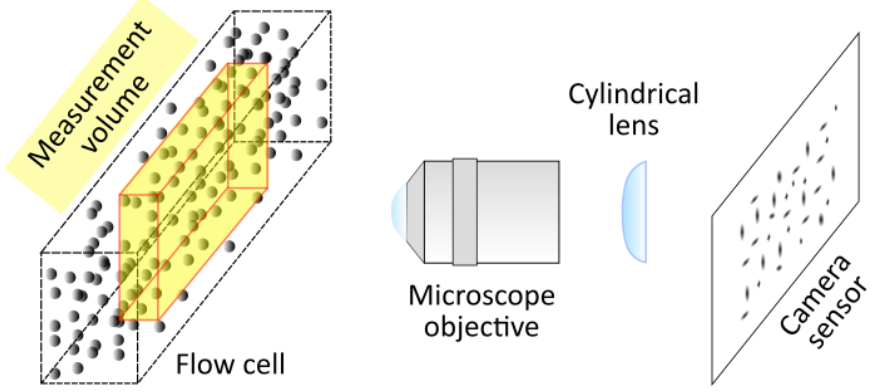

Figure 1: Astigmatic optical system. The measurement volume (shown in yellow) is imaged onto the camera sensor. It is a slice of the total depth of the microfluidic device.

these approaches, the general defocusing particle tracking method $^{26}$ determines the out-of-plane coordinate by crosscorrelating the particle images with a set of training images recovered from known out-of-plane locations.

In this study we extend methods which parameterize particle images obtained by an astigmatic optical system. ${ }^{33-34}$ We describe the particle images using a multi-parameter model rather than solely the diameters of the major and minor axes. In contrast to the cross-correlation approach, ${ }^{26}$ our objectdetection algorithm can distinguish between overlapping particle images, which could allow for higher particle seedingdensities and therefore more detailed flow measurements. We demonstrate this method in a laminar flow through a rectangular 3D channel for which an analytical solution is known. We emphasize its ability to resolve the flow field in all three dimensions despite the use of standard bright-field illumination. This results in significantly lower signal-to-noise (SNR) ratios as compared to the studies we referred to earlier; however, it comes with the added benefit of a wider applicability at a significantly reduced cost as the technique does not require a high-power laser or multiple cameras.

\section{Methods}

\section{Optical modification of particle images}

In this study we employ an astigmatic optical system comprising a microscope objective and a cylindrical lens (see Figure 1). Detailed descriptions of similar optical configurations can be found in the literature. ${ }^{33,36}$ These optical components create unique particle-images depending on the distance from, and position relative to the focal plane, by distorting the round particle-images into elliptical shapes. Behind the focal plane, the major axis of the ellipse is oriented vertically with respect to the camera sensor, whereas ahead of the focal plane it is oriented horizontally. Particle images close to the focal plane form a hybrid, star-like shape (see Figure 2 and Ref. 26). This unique change in particle images along the $z$ direction can be employed to infer the $z$ location of a particle based on its image. 


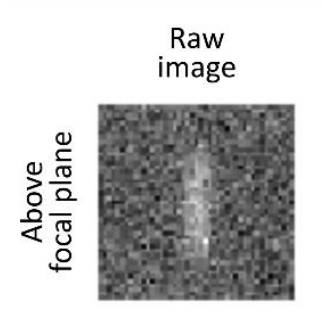

Filtered image
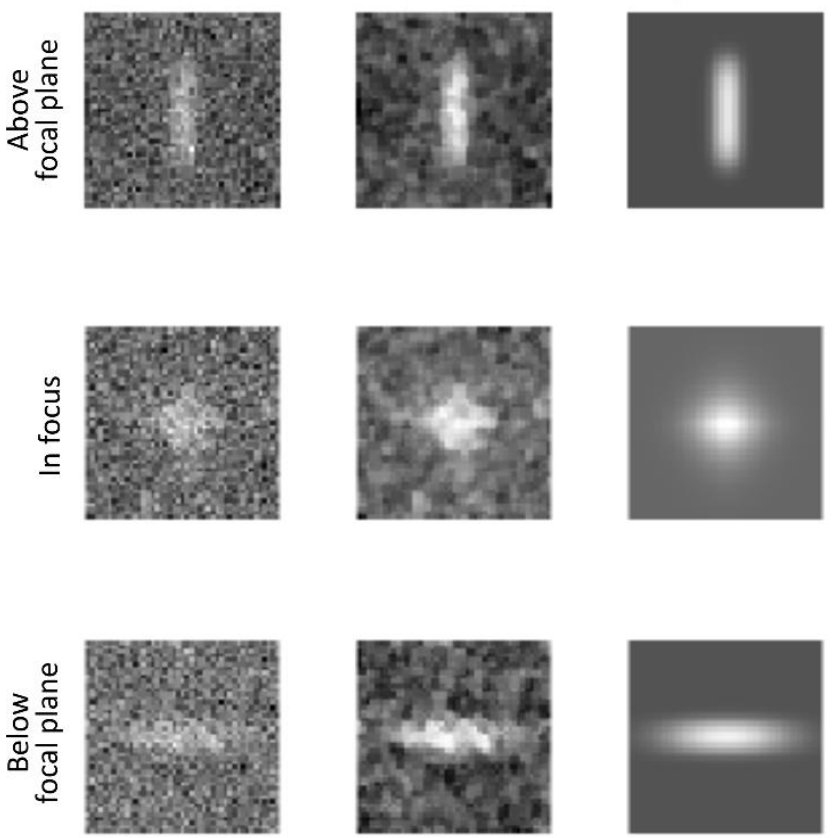

Figure 2: Left column: Examples of particle images at different locations with respect to the focal plane. Middle column: Filtered particle images. Right column: Generalized Gaussian distribution fitted to the filtered particle image. The greyscale corresponds to the inverse of the light detected at the camera sensor. The image size is $45 \times 45$ pixel.

\section{Feature extraction}

To date, two approaches for calibrating astigmatic optical systems have been developed. The most recent one, referred to as generalized defocusing particle tracking velocimetry ${ }^{26}$, compares particle images with a stack of calibration images using cross-correlation. This method allows for arbitrary particle-image shapes but is limited when the particle images overlap. The second calibration approach uses the major and minor axes diameters of the particle images as inputs, $27,36,37,38$ but ignores all other features, despite the fact that they could be used to describe the particle images in more detail.

To address these shortcomings, we begin by fitting a 2D, generalized Gaussian distribution to each particle image:

$$
f(x, y)=\alpha \cdot e^{-\left(\frac{\left|x-x_{\text {cent }}\right|}{\rho_{x}}\right)^{\beta_{x}}-\left(\frac{\left|y-y_{\text {cent }}\right|}{\rho_{y}}\right)^{\beta_{y}}} .
$$

The parameter $\alpha$ describes the maximum intensity of the particle image, $\beta_{x}$ and $\beta_{y}$ are measures of convexity/concavity in the $x$ and $y$ directions, $\rho_{x}$ and $\rho_{y}$ are scale parameters in the $x$ and $y$ directions and describe the spread of the distribution, and $x_{\text {cent }}$ and $y_{\text {cent }}$ are the locations of the centre of the distribution.

This distribution provides a good fit to both the ellipticallyshaped particle images distal to the focal plane, as well as the star-shaped particle images proximal to the focal plane (see, Figure 2). The fitting parameters provide us with a quantitative description of each particle image, and can therefore be used to determine its $z$-location.

An important attribute of this approach is that the particleimage features can be extracted even when two or more particle-images overlap, as long as these are detected as separate objects. In practice, this means that the two particles must produce distinct peaks; however, any off-peak sections of the particle images can still overlap. Another advantage of our fitting tactic is that the $x$ and $y$-coordinates of the particles are included as additional parameters. This allows for the determination of those locations with sub-pixel accuracy.

The trust region algorithm is used as fitting scheme with starting points of $0.01,2$ and 0.2 for parameters $\beta, \rho$ and $\alpha$ respectively. Adding appropriate lower and upper bounds for the fitting parameters improved the robustness and the speed of the procedure. The fitting procedure of one generalized Gaussian distribution took between 0.05 and 0.1 seconds to calculate.

\section{Application}

\section{Experimental apparatus}

To validate our methodology, we examine a laminar channelflow which allows us to compare our velocity-measurements to an analytical solution of the flow field. The dimensions of the flow cell are 3, 7 and $30 \mathrm{~mm}$ in height, width and length, respectively. The cell is made of borosilicate glass (Duran borosilicate glass 3.3, CM Scientific), which matches the refractive index of the injected fluid (Glycerol, 99.5\%, Sigma Aldrich). ${ }^{39}$ The fluid is doped with polystyrene microspheres of $2 \mu \mathrm{m}$ mean diameter (microParticles $\mathrm{GmbH}$ ) at a concentration of $5 \times 10^{-5} \mathrm{w} / \mathrm{v}$.

Imaging is conducted with an inverted microscope (Leica DMi8) comprising a $5 x$ objective (Leica HC PL FLUOTAR $5 x / 0.15$ ) and a cylindrical lens (Thorlabs plano-convex round cylindrical Lens, $f=250 \mathrm{~mm}$ ) housed inside a $2.5 \mathrm{x}$ camera adapter (Leica 10446175). Images are acquired with brightfield illumination, and thus, we recover the shadows of the particles. The camera we employ is a Phantom Lab340 with a resolution of $1440 \times 2560$ pixels at a frame rate of $24 \mathrm{fps}$. The exposure time is set to $550 \mu$ s during flow experiments.

The SNR of our particle images was determined according to the definitions of signal and noise of particle images in the literature. ${ }^{40}$ The mean and variance of the SNR were 5.6 and 0.44 for particles close to the focal plane, which is an order of magnitude lower than the SNRs obtained with pulsed-laser illumination. ${ }^{26}$ Yet, as we demonstrate later on, our methodology is still capable of extracting reliable, quantitative velocity-data from within the investigated flow-field. This is one of the main benefits of this method, making it applicable without the use of expensive illumination sources.

Due to inhomogeneous illumination over the field of view of the sensor, a dedicated image-calibration procedure was developed. First, the flow cell was filled with glycerol, and images with background illumination (and no particles) were collected as white-references. Then the brightness of the illumination was lowered and black-reference images were recorded. This simulates the maximum shade a particle could cast at each pixel. A linear interpolation between the white and the black reference is used to calibrate each pixel. The intensity distribution of the background illumination varies with the focal position of the microscope objective, and thus, separate calibration images were recorded at all relevant focal 


\section{Feature extraction from overlapping particle images}
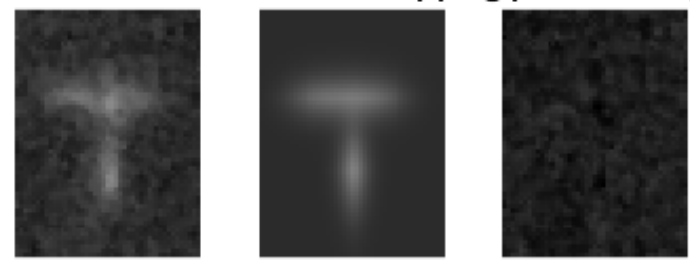

Particle images Fitted distributions

Residuals

Figure 3: Example of overlapping particle images

planes. This process also removes any images of dirt particles that may be present at the walls of the cell.

\section{Generating the training dataset}

To develop a mathematical relation between the fit parameters and the particle $z$-locations, a calibration dataset was acquired. This dataset contains a set of fit parameters based on the geometries of the observed particle images at known locations in the $z$-direction. The dataset accounts for all factors which influence the particle-image features: the location on the $z$-axis, the $x-y$ coordinates and noise of the camera sensor. Fit parameters have to be evaluated based on all these variables in order to allow for a robust prediction of the $z$-location. The training images were acquired under the same conditions as the flow measurements. ${ }^{26}$

After imaging the flow cell with pure glycerol, the cell is flooded with particle-doped glycerol until a homogeneous distribution of particles is observed over the depth of the channel. After allowing the fluid to settle for 1 hour, the acquisition of calibration images commenced. Calibration images were acquired at a total of 1000 slices along the entire depth of the flow channel $(3 \mathrm{~mm})$, with the distance between adjacent slices set to $3 \mu \mathrm{m}$. At each slice, a total of 4 images were collected to account for the noise of the camera sensor. The investigated volume was located in the centre of the rectangular flow cannel and spanned 2048, 1152 and $3000 \mu \mathrm{m}$ in length, width and depth respectively. Thus, it did not cover the entire width of $7 \mathrm{~mm}$ of the channel.

\section{Object detection \& feature extraction}

The training images from each slice were averaged to reduce camera noise. The averaged images were then scaled with the black and white reference signals, by subtracting the white reference (background) and dividing by the difference between the white and the black reference. For easier processing, the resulting images were inversed, causing the particles to appear bright and the background dark. The inverted images were smoothed by four different 2D median filters of sizes [3,5], [5,3], [1,3] and [3,1] pixels. The filters were applied separately and the results were averaged. This process enhances the SNR of the particle images along the $x$ and $y$ axes. Finally, local peaks in the images were identified by a dedicated algorithm that employs additional internal filtering and thresholding steps. ${ }^{41}$

All identified peaks are considered candidate particle images. Any peaks detected in close vicinity were scrutinised further in order to determine if they were individual particles or multiple

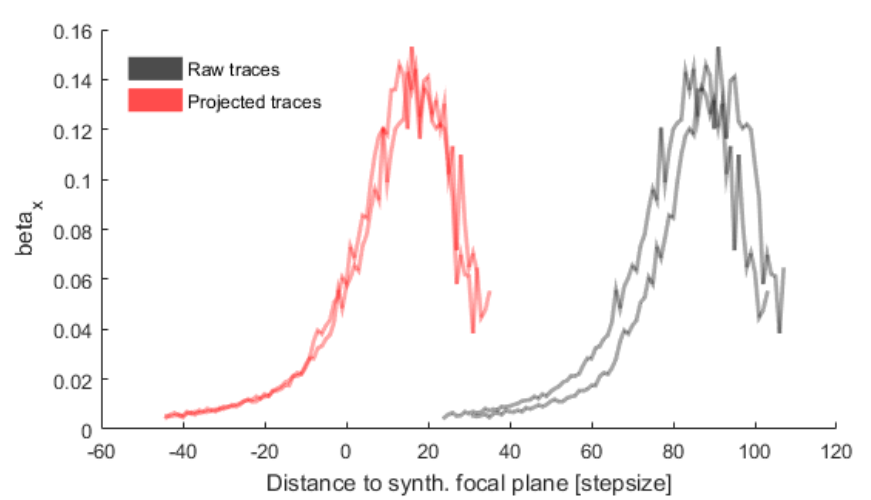

Figure 4: Example for the projection of two raw traces (grey) onto one common synthetic plane. Here, only parameter $\beta_{x}$ of two traces is shown for simplicity; however, the RMSE was minimised between all parameters and all traces.

peaks corresponding to a single particle. This step was implemented by iteratively increasing the number of generalized Gaussian distributions which were fitted to the examined group of peaks. Once the root mean square error (RMSE) between the particle image and the fit fell to the noise level within the same image, the fitting process was terminated and any excessive peaks were deleted. This iterative procedure removed multiple peaks per particle and the remaining peaks were considered to be unique particles. Finally, if any particles were found within a distance of 25 pixels or less from each other, their signal distributions were considered to impact each other's fitting process. In these cases, the parameters of both particle images were cooptimised within the same fitting task. This allowed for the extraction of image features from particle images which are slightly overlapping (see Figure 3 ). Each particle image has to produce a distinctive peak, which can be detected by the initial peak detection algorithm. If this is not the case the iterative procedure might only fit one distribution to two particle images. A particle rejection scheme could be easily implemented by comparing extracted image parameters to the image parameters in the training dataset and thresholding the maximum allowed distance in parameter space.

We used a low seeding density of particles which produced few overlapping particle images to focus this work on the impact of the additional image features on the calibration. Less than 1 in 30 frames contained an overlapping particle image pair. Therefore, overlapping particle images were ignored when determining the uncertainty of the method. When employing this method with a higher particle density, additional errors from co-optimising a larger number of image parameters should be investigated in more detail as well as the maximum possible degree of particle overlapping.

\section{Aligning particles on a common synthetic plane}

The fitting parameters extracted from the particle images of the calibration-image stack have to be mapped to the locations of the particles with respect to the focal plane. To that end, particle-traces are constructed along the stack of training images. A trace comprises a sequence of fitting parameter values from the images of a single particle over all frames where the particle is visible. Thus, a trace describes how the image (i.e. the set of fitting parameters) of a single particle 
Feature weights based on neighborhood component analysis

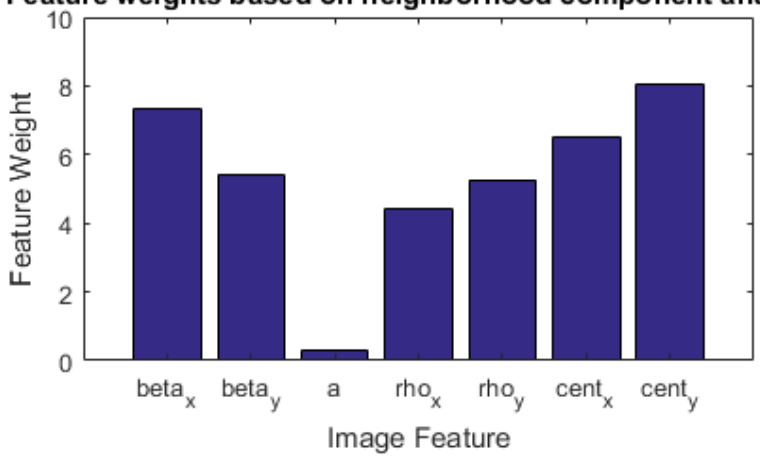

Figure 5: Feature weights based on neighborhood component analysis. It can be seen that the amplitude of the distribution (parameter a) contains little information with regards to the $z$-value.

changes with the $z$-coordinate of that particle. To evaluate the whole parameter space and to account for noise, the training dataset comprises a large number of traces. Only traces of particles which span a minimum of 75 successive slices $(\hat{=} 225$ $\mu \mathrm{m})$ are included for further analysis.

A priori, the $z$-locations of the particles of the traces are unknown, as the particles are randomly distributed within the flow channel. So, initially, traces consist only of a sequence of fitting parameters. To add spatial information to the traces, the indices of the traces are multiplied by the step size which was used during the calibration. This allows for the fitting parameters within one trace to be related to each other and creates a first estimate for the $z$-location of each element within a trace.

To find a spatial relation between two traces the following procedure was implemented. The traces of the fitting parameters of each particle create a similar signal with respect to their out-of-plane coordinate. Hence, the traces can be

Table 1: Comparison of candidate models by their performance (RMSE) with regards to the training dataset

\begin{tabular}{|c|c|c|c|}
\hline Model & Hyper-parameter & Value & $\begin{array}{l}\text { RMSE } \\
{[\mu \mathrm{m}]}\end{array}$ \\
\hline $\begin{array}{l}\text { Fully Connected } \\
\text { Neural Network }\end{array}$ & $\begin{array}{l}\text { Number of } \\
\text { Neurons / Layer } \\
\text { Number of Layers }\end{array}$ & $\begin{array}{l}9 \\
3\end{array}$ & 6.15 \\
\hline $\begin{array}{l}\text { Boosted } \\
\text { regression tree } \\
\text { ensemble }\end{array}$ & $\begin{array}{l}\text { Maximum number } \\
\text { of splits } \\
\text { Number of trees } \\
\text { Learning rate }\end{array}$ & $\begin{array}{l}10 \\
5000 \\
0.1\end{array}$ & 7.11 \\
\hline $\begin{array}{l}\text { K-nearest } \\
\text { neighbour }\end{array}$ & $\begin{array}{l}\theta \\
k\end{array}$ & $\begin{array}{l}3.7 \\
10\end{array}$ & 10.79 \\
\hline $\begin{array}{l}\text { Ensemble model } \\
\text { equal weights }\end{array}$ & & & 6.74 \\
\hline $\begin{array}{l}\text { Ensemble model } \\
\text { adjusted weights }\end{array}$ & & & 6.05 \\
\hline $\begin{array}{l}\text { Boosted } \\
\text { regression tree } \\
\text { ensemble - } \\
\text { "conventional" } \\
\text { parameters }\end{array}$ & $\begin{array}{l}\text { Maximum number } \\
\text { of splits } \\
\text { Number of trees } \\
\text { Learning rate }\end{array}$ & $\begin{array}{l}14 \\
5000 \\
0.1\end{array}$ & 22.1 \\
\hline
\end{tabular}

projected onto one common plane by shifting traces relative to each other on the z-axis such that the RMSE between them is minimized. After all traces are aligned relative to each other, they are shifted by one constant value to bring the centre of the traces closer to the focal plane of the microscope. An illustrative example of this procedure, projecting two raw traces onto one common plane, is shown in Figure 4.

A first coarse $z$-shift was conducted by cross-correlating each trace with respect to all other traces on the parameters $\alpha, \beta_{x}$ and $\beta_{y}$. This allowed for a projection of the traces onto a common plane with a resolution given by the step size between two consecutive image slices $(3 \mu \mathrm{m})$. The $z$-shift of each trace with respect to the synthetic plane was subsequently refined by shifting the functions of the feature values with respect to $z$. Linear interpolation between feature values at imaged slices allowed for a sub-slice resolution shift along the $z$-axis. This shift was determined numerically by minimising the RMSE of each trace with respect to all other traces. The projection of all traces onto one common plane yielded a complete training dataset. At this point, the image features were linked to a common plane, with a constant offset to the real focal plane of the microscope.

A neighborhood component analysis on the training dataset reveals that the only parameter which contains little information about the z-location is the amplitude of the generalized Gaussian distribution (see Figure 5). This confirms results from conventional calibration approaches, where only parameters $\rho_{x}, \rho_{y} x_{\text {cent }}$ and $y_{\text {cent }}$ have previously been used and parameter $\alpha$ was ignored. In addition, the results of the analysis shows the value of the parameters $\rho_{x}$ and $\rho_{y}$, which contain significant predictive information.

\section{Model development}

After generating the training dataset, a function $g$ was found, relating the fitting parameters and the $z$-location of the respective particles:

$$
g\left(\alpha, \beta_{x}, \beta_{y}, \rho_{x}, \rho_{y}, x_{\text {cent }}, y_{\text {cent }}\right)=z
$$

In addition to the fitting parameters, the location parameters $x_{\text {cent }}$ and $y_{\text {cent }}$ can be employed to increase the precision of the predictive algorithm. This is due to the lateral aberration of the optics which cause slight modifications of the particle images depending on the in-plane locations $x_{\text {cent }}$ and $y_{\text {cent }}$. This function can be approximated with any flexible regression model. Therefore, MATLAB implementations of three different models were tested: (i) an adapted k-nearest neighbour model, (ii) a neural network and (iii) an ensemble of boosted regression trees. The neural network and boosted regression tree ensemble were applied without modifications. Hyperparameters of the models were tuned using 10-fold cross validation to find model configurations with the lowest possible generalization error (see Table 1).

The k-nearest neighbour model was adapted in order to avoid bias arising from non-uniform sampling of the parameter space along the $z$-axis. Close to the focal plane, the SNR is higher and particles are more likely to be detected. Further away from the focal, plane, the particle detection algorithm does not always detect particles, and thus, this region is more sparsely sampled. This aspect creates two challenges. 
Firstly, when selecting the k-nearest neighbours, it becomes more probable to include neighbours which are located closer to the focal plane. This leads to a bias in $z$-predictions towards values which are closer to the focal plane. This challenge can be addressed by weighing the training data points at locations $z_{i}$ with respect to the probability density of detected particles at their $z$-location $w\left(z_{i}\right)$. This levels out the bias in z-prediction towards the focal plane.

The second challenge associated with sparse sampling away from the focal plane is that with fewer data points, it becomes more probable to include a neighbour with a larger distance in parameter space. This is undesired since those neighbours correspond to images which differ more than the images of neighbours which are closer in parameter space. This can be accounted for by lowering the weights of neighbours depending on their Euclidean distance in parameter space $d_{i}\left(\alpha, \beta_{x}, \beta_{y}, \rho_{x}, \rho_{y}, x_{\text {cent }}, y_{\text {cent }}\right)$. We therefore applied an exponential kernel $d_{i}^{\theta}$ to the k-nearest neighbours at locations $z_{i}$.

$$
g\left(\alpha, \beta_{x}, \beta_{y}, \rho_{x}, \rho_{y}, x_{\text {cent }}, y_{\text {cent }}\right)=\frac{\sum_{i}^{k} \frac{z_{i}}{w\left(z_{i}\right)_{i} d_{i}^{\theta}}}{\sum_{i}^{k} \frac{1}{w\left(z_{i}\right)_{i} d_{i}^{\theta}}}
$$

In addition to the three individual models, two model ensembles were evaluated as well. The first ensemble simply averages the predictions of the three individual models and the second uses adjusted weights for each individual model. The optimal weights for each individual model were determined, once more, using 10 -fold cross validation. They were found to be $0.750,0.214$ and 0.036 for the neural network, boosted regression tree ensemble and k-nearest neighbour model respectively.

Table 1 shows that the neural network has the lowest crossvalidation RMSE in the training dataset. Yet, the ensemble with adjusted weights outperforms the neural network. This is not surprising given that the individual models are inherently very different. ${ }^{42}$ Based on this comparison, the ensemble model with adjusted weights was considered the most suitable

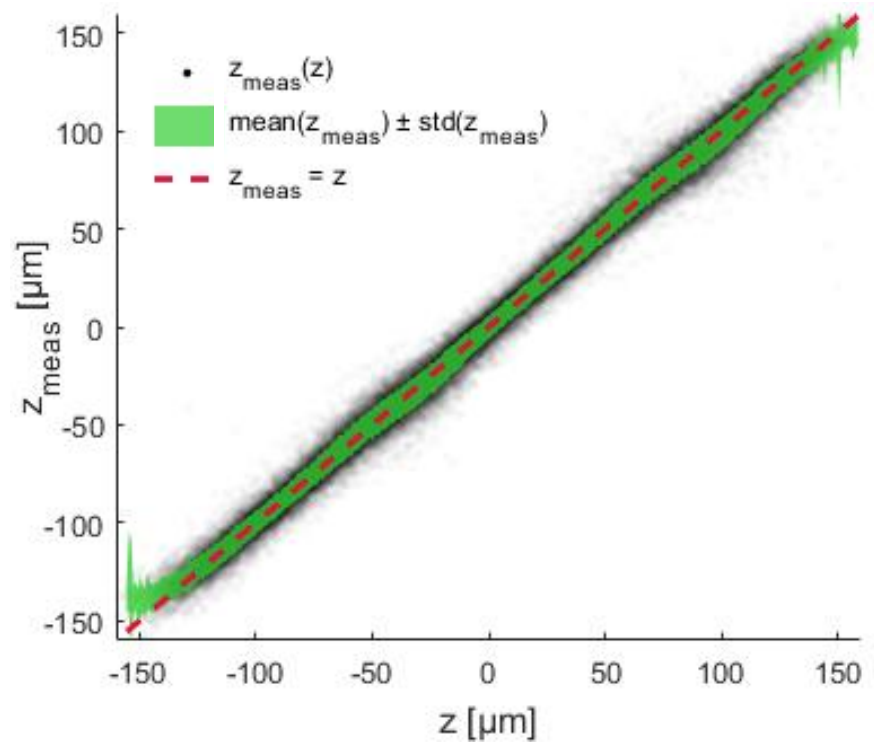

Figure 6: 10 fold cross-validated measured z-locations using the boosted regression tree ensemble compared to real $z$ location. Green area covers the mean \pm the standard deviation of the measured $z$ location at a given true $z$ location algorithm. However, when applied to the data from the flow experiment, the boosted regression tree ensemble performed best in comparison to all other models and model ensembles. This might be due to a mismatch between the high-quality training data and the lower quality data from the flow experiment. Therefore, the final model selection and error analysis were performed based on the results of the experiment (i.e. the comparison between the measured and analytically calculated velocities).

An additional boosted regression tree model was trained and hyperparameter-optimized using only the parameters $\rho_{x}, \rho_{y}, x_{\text {cent }}$ and $y_{\text {cent }}$. This simulates the performance of these previously used "conventional" image parameters. The poor cross-validated rmse of $22.1 \mu \mathrm{m}$ confirms the results of the neighbourhood component analysis. This suggests that there is significant information about the $z$-location encoded in the additional parameters of the generalized Gaussian distribution, which is the reason for the better performance of all models trained with all image parameters.

Figure 6 shows how the measured z-locations relate to the real $z$-locations. The $z$ locations were measured in the respective hold out datasets of a 10-fold cross-validation of the boosted regression tree ensemble. Above a distance of approximately $120 \mu \mathrm{m}$ from the focal plane the model becomes unreliable. Here, the measured z-locations have a bias towards the focal plane and a significantly higher variance.

\section{Flow measurements}

Particle velocities were measured at flow rates of 1.0 and 2.5 $\mathrm{mL} / \mathrm{h}$, and images were captured at a rate of 24 frames per second. Particle sedimentation between frames is $5 \times 10^{-5} \mu \mathrm{m}$, the Reynolds number for both experiments is $<<1$ and the Peclet number is 37 . Thus, enabling the $2 \mu \mathrm{m}$ polystyrene particles as tracers of laminar flow. Due to the higher errors at a distance above $120 \mu \mathrm{m}$ from the focal plane, particles could only be tracked within a slice of roughly $240 \mu \mathrm{m}$ thickness. To measure through the whole depth of the flow cell $(3000 \mu \mathrm{m})$, a stack of image sets was acquired at focal positions $150 \mu \mathrm{m}$ apart from each other. Each individual image set contained 452 sequential images. Acquisition speed in the z-direction might be limited by the speed of the microscope stage or the synchronisation of the microscope components. The employed Leica closed loop microscope stage can move between focal planes within $30 \mathrm{~ms}$.

From each flow image, particle fitting parameters were extracted and the particle locations were determined using all candidate models. The particles were tracked between successive frames using the Hungarian Method. ${ }^{42}$ Trajectories with particles visible at less than 100 time steps were rejected. This threshold was found by analysing the relationship between the standard deviation of the z-locations of a trace and the amount of time steps in which it is visible. In traces with less than 100 time step visibility, the standard deviation of $z$ locations is significantly larger, which would add noise to the measured velocity field. Slightly more $(=893)$ trajectories were analysed in the experiment when the flow rate was set to $1.0 \mathrm{~mL} / \mathrm{h}$, as compared to 827 trajectories in the experiment with a flow rate of $2.5 \mathrm{~mL} / \mathrm{h}$. 
For a quantitative description of the overall flow field, average velocities were calculated for each particle trajectory. The measured velocities approach zero near the walls (in the $z$ direction of the flow) and peak in the middle of the flow cell. For a quantitative comparison between the measured and the calculated flow velocities, an analytical solution for the velocity profile was determined. ${ }^{44}$

Velocity measurements were recovered using all three models and the two ensemble versions and were compared to analytical calculations by determining mean relative deviations. The ranking of the models differs compared to the training dataset. With a mean relative error of $3.3 \%$ for the velocity in the $x$-direction, the boosted regression tree ensemble was the best performing model on the flow dataset. Relative errors have been determined by relating the absolute errors to the velocity of the analytical solution at the corresponding $z-y$ location. All further error analysis is based on the performance of the boosted regression tree ensemble model as it was the best performing model with respect to the analytical solution of the flow experiments.

A comparison of the displacements that were recovered with the analytical solution is shown in Figure 7 . The measurements do not show any obvious bias when compared to the analytical solution. For these trajectory-averaged displacements the mean relative errors in the $x$-direction are $3.7 \%$ and $2.9 \%$ for the $1.0 \mathrm{~mL} / \mathrm{h}$ and the $2.5 \mathrm{~mL} / \mathrm{h}$ experiments respectively.

Previously reported relative errors of the displacement in the x-direction are $0.9 \%{ }^{27}$ as well as between $1 \%$ and $6 \%{ }^{26}$ for experiments in smaller domains of 100 and $200 \mu \mathrm{m}$ height. These errors relate to single displacement measurements instead of interpolated measurements. We therefore determined relative and absolute errors for non-smoothed displacement measurements between single time steps in our
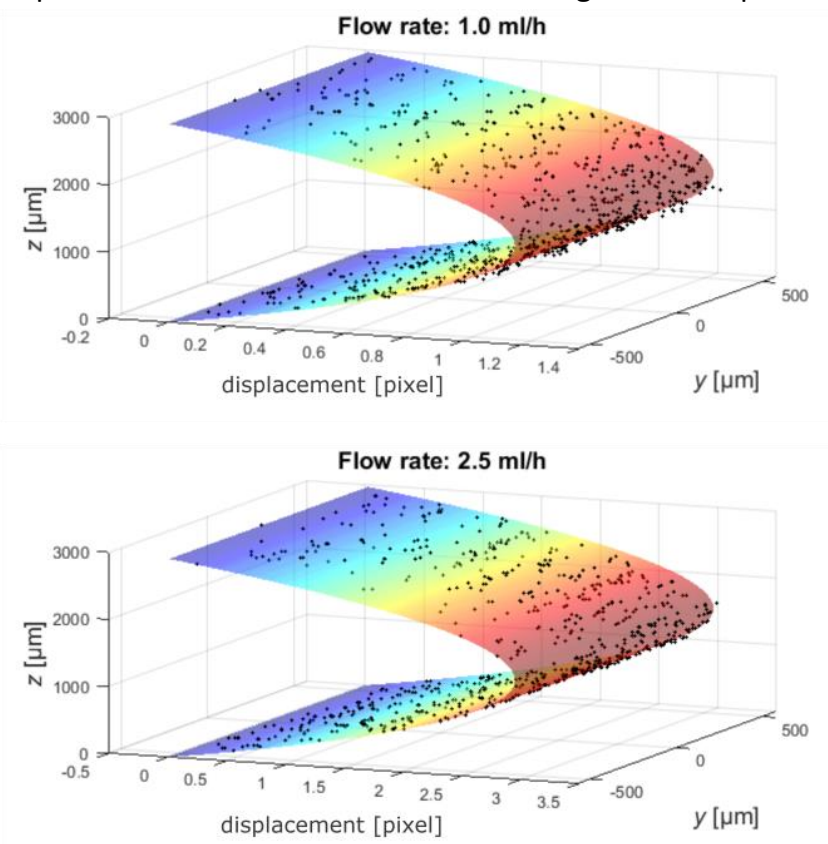

Figure 7: Comparison of the measured average displacement in the $x$-direction of each trajectory with the analytical solution of the flow field for both experiments. The model used for these measurements is the boosted regression tree ensemble which had the smallest error as compared to the analytical solution of the flow field.

1 pixel $\triangleq 0.8 \mu \mathrm{m}, 1$ frame $\triangleq 1 / 24 \mathrm{~s}$ dataset. The displacement errors for the two experiments were estimated by calculating the standard deviation of residuals between analytical and measured displacements. They were found to be 0.21 and 0.22 pixels corresponding to velocity errors of $4.0 \mu \mathrm{m} / \mathrm{s} 4.2 \mu \mathrm{m} / \mathrm{s}$. Scaled by the maximum velocity of the flow this translates into relative errors of $17.8 \%$ and $7.3 \%$. A higher experimental velocity reduces the relative error in the $2.5 \mathrm{ml} / \mathrm{h}$ experiment by more than half, while the absolute error increases by only $10 \%$. Relative errors are significantly higher in this study $(7.3-17.8 \%)$ than the errors in the literature $(0.9-6 \%)^{26,27}$. Displacement errors are roughly double in our experiments as compared to errors in the literature (0.1 pixel) ${ }^{26,27}$.

The lower displacement and relative errors in the literature are likely to be linked to the significantly higher SNRs obtained in these studies. Previous studies employed cameras with CCD/sCMOS sensors and pulsed lasers for illumination ${ }^{26,27}$ instead, we acquired images with a basic microscope arrangement that uses a CMOS sensor, and LED illumination. This explains why the reported level of noise of $<=15 \%$ in literature ${ }^{27}$ is well below the noise level of $\sim 80 \%$ (based on their definition of the noise level) in our images. The same is true for the value of the SNR which is in the range of $5-42$ in a previous study ${ }^{26}$ as compared to a mean value of 5.7 in this study. Despite the differences in image quality, the method presented here is still capable of describing the flow field at the scale at which the study was conducted.

The displacement error does not show any trends throughout the entire depth of measurement for both experiments (see Figure 8). We purposely measured as close to the boundaries at the top and at the bottom of the flow cell as possible, as many research areas are particularly interested in near-wall measurements. The constant displacement error along the zaxis suggests that the solid-liquid boundaries do not impact the accuracy of the measurement system significantly. This is in agreement with a previous study where the same behaviour was reported. ${ }^{8}$ This suggests that the method proposed in the present work might be suitable for investigating flows in porous media where solid-liquid interfaces are encountered frequently.

\section{Conclusions}

The presented defocusing particle tracking method provides a low cost, yet reliable alternative to observe $3 \mathrm{D}$ flow
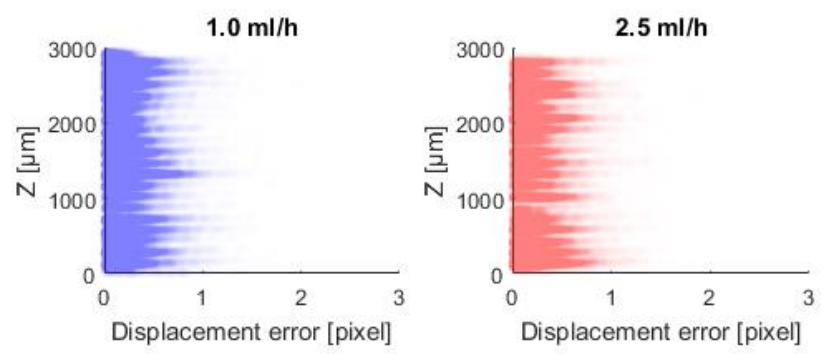

Figure 8: Single errors between measured particle displacement and analytical solution. Relative errors are relative to the maximum velocity of the analytical solution. 1 pixel $=0.8 \mu \mathrm{m}, 1$ frame $=1 / 24 \mathrm{~s}$ 
phenomena at the pore-scale. It is based on a simple calibration approach to track particles in a 3D fluid flow using an astigmatic optical system. A 2D generalized Gaussian distribution is employed to parameterise particle images. The preparation of a training dataset comprised of these fitting parameters paired with known out-of-plane locations is shown in detail. This proposed workflow can be applied to any transparent microfluidic device. Once the training data is collected, the calibration of the apparatus is purely empirical and can be conducted with a standard machine learning tool for multivariate regression. The method is demonstrated using a brightfield illumination. This confirms the robustness of the method with regards to the use with images of low signal to noise ratio.

The displacement errors in channel flows with 1.0 and 2.5 $\mathrm{mL} / \mathrm{h}$ were obtained by comparing experimental velocity measurements with analytic solutions for the flow field, and were found to be 0.21 and 0.22 pixel, respectively. Although these values are higher than the errors reported in other studies that employ pulsed lasers for illumination and $\mathrm{CCD} / \mathrm{sCMOS}$ cameras, our work demonstrates that it is possible to conduct 3D particle velocimetry with an apparatus based on a microscope with conventional brightfield illumination and a CMOS sensor. This makes the method accessible to a larger community.

We validated the method in a laminar channel flow for which an analytical solution is known. In the future we intend to employ this method to investigate single and multiphase flows in porous media with matched refractive index. The flow phenomena that we seek to investigate span length scales in the order of 10 s of $\mu \mathrm{m}$ to a few $\mathrm{mm}$ in all three dimensions. Therefore, we showed how measurements at multiple focal levels can be combined to cover large measurement depths. Imaging at multiple focal levels comes at the cost of moving the microscope stage during the experiment. In our case we investigated a volume with dimensions $2.0,1.2$ and $3.0 \mathrm{~mm}$ in length, width and depth respectively.

\section{Conflicts of interest}

"There are no conflicts to declare".

\section{Acknowledgements}

We gratefully acknowledge funding from the Qatar Carbonates and Carbon Storage Research Centre (QCCSRC), provided jointly by Qatar Petroleum, Shell, and the Qatar Science \& Technology Park. This work was also supported by the UK Engineering and Physical Sciences Research Council (EPSRC) [grant numbers EP/K008595/1 and EP/L020564/1]. Data supporting this publication can be obtained on request from cep-lab@imperial.ac.uk.

\section{References}

1 M. Boon, B. Bijeljic and S. Krevor, Observations of the impact of rock heterogeneity on solute spreading and mixing, Water Resour. Res, 2017, 53, 4624-4642.

2 M. Boon, B. Bijeljic, B. Niu and S. Krevor, Observations of 3-D transverse dispersion and dilution in natural consolidated rock by X-ray tomography, Adv. Water Resour., 2016, 96, 266-281.

3 H. P. Menke, C. A. Reynolds, M. G. Andrew, J. P. Pereira Nunes, B. Bijeljic and M. J. Blunt, 4D multi-scale imaging of reactive flow in carbonates: Assessing the impact of heterogeneity on dissolution regimes using streamlines at multiple length scales, Chem. Geol., 2018, 481, 27-37.

4 H. P. Menke, M. G. Andrew, M. J. Blunt and B. Bijeljic, Reservoir condition imaging of reactive transport in heterogeneous carbonates using fast synchrotron tomography - Effect of initial pore structure and flow conditions, Chem. Geol., 2016, 428, 15-26.

5 S. Klump, O. A. Cirpka, H. Surbeck and R. Kipfer, Experimental and numerical studies on excess-air formation in quasisaturated porous media, Water Resour. Res, 2008, 44, W05402.

6 C. Chang, Q. Zhou, M. Oostrom, T. J. Kneafsey, H. Mehta, Pore-scale supercritical $\mathrm{CO} 2$ dissolution and mass transfer under drainage conditions, Adv. Water Resour., 2017, 100, 14-25.

7 S. Berg, H. Ott, S. A. Klapp, A. Schwing, R Neiteler, N. Brussee, A. Makurat, L. Leu, F. Enzmann, J.-O. Schwarz, M. Kersten, S. Irvine and M. Stampanoni, Real-time 3D imaging of Haines jumps in porous media flow, PNAS, 2013, 110, 3755-3759.

8 S. S. Datta, J. B. Dupin, D. A. Weitz, Fluid breakup during simultaneous two-phase flow through a three-dimensional porous medium, Phys. Fluids, 2014, 26, 062004.

9 M. Holzner, V. L. Morales, M. Willmann, and M. Dentz, Intermittent Lagrangian velocities and accelerations in threedimensional porous medium flow, Phys. Rev. E., 2015, 92, 013015.

10 M. Raffel, C. E. Willert, S. T. Wereley, and J. Kompenhans, Particle Image Velocimetry: A Practical Guide, Experimental Fluid Mechanics, Springer, Berlin, 2007.

11 C. J. Kähler, S. Scharnowski and C. Cierpka, On the uncertainty of digital PIV and PTV near walls, Exp. Fluids, 2012, 52, 1641.

12 A. Charogiannis, J. S. An, and C. N. Markides, A simultaneous planar laser-induced fluorescence, particle image velocimetry and particle tracking velocimetry technique for the investigation of thin liquid-film flows, Exp. Therm. Fluid Sci., 2015, 68, 516.

13 A. Charogiannis, F. Denner, B. G. M. van Wachem, S. Kalliadasis, and C. N. Markides, Statistical characteristics of falling-film flows: A synergistic approach at the crossroads of direct numerical simulations and experiments, Phys. Rev. Fluids, 2017, 2, 014002.

14 I. Zadrazil and C. N. Markides, An experimental characterization of liquid films in downwards co-current gasliquid annular flow by particle image and tracking velocimetry, Int. J. Multiphase Flow, 2014, 67, 42-53.

15 R. G. Morgan, C. N. Markides, C. P. Hale, G. F. Hewitt, Horizontal liquid-liquid flow characteristics at low superficial velocities using laser-induced fluorescence, Int. J. Multiphase Flow, 2012, 43, 101-117.

16 P. Thurgood, S. Baratchi, C. Szydzik, A. Mitchella and K. Khoshmanesh, Porous PDMS structures for the storage and release of aqueous solutions into fluidic environments, $L a b$ Chip, 2017, 17, 2517.

17 H. Gong, B. P. Bickham, A. T. Woolley and G. P. Nordin, Custom 3D printer and resin for $18 \mu \mathrm{m} \times 20 \mu \mathrm{m}$ microfluidic flow channels, Lab Chip, 2017, 17, 2899.

18 Y. Cheng, Time-Efficient High-Resolution Large-Area NanoPatterning of Silicon Dioxide, Micromachines, 2017, 8, 59. 
19 H. B. Evans, S. Gorumlu, B. Aksak, L. Castillo and J. Sheng, Holographic microscopy and microfluidics platform for measuring wall stress and 3D flow over surfaces textured by micro-pillars, Sci. Rep., 2016, 6, 28753.

20 K. Xu, T. Liang, P. Zhu, P. Qi, J. Lu, C. Huha and M. Balhoffa, A 2.5-D glass micromodel for investigation of multi-phase flow in porous media, Lab Chip, 2017, 17, 640.

21 M. Rücker, S. Berg, R. T. Armstrong, A. Georgiadis, H. Ott, A. Schwing, R. Neiteler, N. Brussee, A. Makurat, L. Leu, M. Wolf, F. Khan, F. Enzmann, M. Kersten, From connected pathway flow to ganglion dynamics, Geophys. Res. Lett., 2015, 42, 3888-3894.

22 C. A. Reynolds, H. Menke, M. Andrew, M. J. Blunt and S. Krevor, Dynamic fluid connectivity during steady-state multiphase flow in a sandstone Dynamic fluid connectivity during steady-state multiphase flow in a sandstone, PNAS, 2017, 114, 8187-8192.

23 Y. Bachmat, J. Bear, On the Concept and Size of a Representative Elementary Volume (Rev), Advances in Transport Phenomena in Porous Media, 1987, 128, 3-22.

24 P. Mostaghimi, M. J. Blunt, B. Bijeljic, Computations of Absolute Permeability on Micro-CT Images, Math. Geosci., 2013, 45, 103-125.

25 F. Pereira, M. Gharib, D. Dabiri and D. Modarress, Defocusing digital particle image velocimetry: a 3-component 3dimensional DPIV measurement technique. Application to bubbly flows, Exp. In Fluids, 2000, 29, S078.

26 R. Barnkob, C. J. Kähler and M. Rossi, General defocusing particle tracking, Lab Chip, 2015, 15, 3556-3560.

27 C. Cierpka, M. Rossi, R. Segura and C. J. Kähler, On the calibration of astigmatism particle tracking velocimetry for microflows, Meas. Sci. Technol., 2011, 22, 015401.

28 S. S. Datta, H. Chiang, T. S. Ramakrishnan, D. A. Weitz, Spatial Fluctuations of Fluid Velocities in Flow through a ThreeDimensional Porous Medium, Phys. Rev. Lett., 2013, 111 064501.

29 H. Kim, J. Westerweel, G. Elsinga, Comparison of Tomo-PIV and 3D-PTV for microfluidic flows, Meas. Sci. Technol. 2013 24, 024007

30 K. Alim, S. Parsa, D. A. Weitz and M. P. Brenner, Local Pore Size Correlations Determine Flow Distributions in Porous Media, Phys. Rev. Lett., 2017, 119, 144501.

31 Y. Edery, S. Berg and D. A. Weitz, Surfactant Variations in Porous Media Localize Capillary Instabilities during Haines Jumps, Phys. Rev. Lett., 2018, 120, 028005.

32 W. H. Tien, Development of multi-spectral three-dimensional micro particle tracking velocimetry, Meas. Sci. Technol., 2016, 27, 084010.

33 C. Cierpka, R. Segura, R. Hain and C. J. Kähler, A simple single camera 3C3D velocity measurement technique without errors due to depth of correlation and spatial averaging for microfluidics, Meas. Sci. Technol., 2010, 21, 045401.

$34 \mathrm{M}$. Wu, J. W. Roberts and M. Buckley, Three-dimensional fluorescent particle tracking at micron-scale using a single camera, Exp. Fluids, 2005, 38, 461-465.

35 E. Afik, Robust and highly performant ring detection algorithm for $3 \mathrm{~d}$ particle tracking using $2 \mathrm{~d}$ microscope imaging, Sci. Rep., 2015, 5, 13584.

36 M. Rossi and C. J. Kähler, Optimization of astigmatic particle tracking velocimeters, Exp. Fluids, 2014, 55, 1809.

37 S. Chen, N. Angarita-Jaimes, D. Angarita-Jaimes, B. Pelc, A. H. Greenaway, C. E. Towers, D. Lin and D. P. Towers, Wavefront sensing for three-component three-dimensional flow velocimetry in microfluidics, Exp. Fluids, 2009, 47, 849.

38 R. Hain, C. J. Kähler, R. Radespiel, Principles of a Volumetric Velocity Measurement Technique Based on Optical Aberrations Article, Imaging Measurement Methods for Flow Analysis, 2009, 106, 1-10.

39 S. F. Wright, I. Zadrazil, C. N. Markides, A review of solidfluid selection options for optical-based measurements in single-phase liquid, two-phase liquid-liquid and multiphase solid-liquid flows, Exp. Fluids, 2017, 58, 108.

40 T. Savin, P. S. Doyle, Static and dynamic errors in particle tracking microrheology, Biophys. J., 2005, 88, 623-638.

41 Nathan, MATLAB Central File Exchange, 2013 https://uk.mathworks.com/matlabcentral/fileexchange/373 88-fast-2d-peak-finder

42 L. Breiman, Stacked regressions, Machine Learning, 1996, 24 49-64.

$43 \mathrm{H}$. W. Kuhn, The Hungarian Method for the Assignment Problem, Naval Research Logistics, 1995, 2, 83-97.

44 W. Rybinski, J. Mikielewicz, Analytical solutions of heat transfer for laminar flow in rectangular channels, Arch. Thermodyn., 2014, 35, 29-42. 